\title{
Some Comments on Rigorous Finite-Volume Euclidean Quantum Field Path Integrals in the Analytical Regularization Scheme
}

\author{
Luiz C. L. Botelho \\ Departamento de Matemática Aplicada, Instituto de Matemática, Universidade Federal Fluminense, \\ Rua Mario Santos Braga, 24220-140 Niterói, RJ, Brazil \\ Correspondence should be addressed to Luiz C. L. Botelho, botelho.luiz@superig.com.br \\ Received 4 March 2011; Accepted 20 April 2011 \\ Academic Editor: Klaus Kirsten \\ Copyright (C) 2011 Luiz C. L. Botelho. This is an open access article distributed under the Creative \\ Commons Attribution License, which permits unrestricted use, distribution, and reproduction in \\ any medium, provided the original work is properly cited. \\ Through the systematic use of the Minlos theorem on the support of cylindrical measures on $R^{\infty}$, \\ we produce several mathematically rigorous finite-volume euclidean path integrals in interacting \\ euclidean quantum fields with Gaussian free measures defined by generalized powers of finite- \\ volume Laplacian operator.
}

\section{Introduction}

Since the result of R.P. Feynman on representing the initial value solution of Schrodinger Equation by means of an analytically time continued integration on a infinite-dimensional space of functions, the subject of Euclidean Functional Integrals representations for Quantum Systems has became the mathematical-operational framework to analyze Quantum Phenomena and stochastic systems as showed in the previous decades of research on Theoretical Physics [1-3].

One of the most important open problems in the mathematical theory of Euclidean Functional Integrals is that related to implementation of sound mathematical approximations to these Infinite-Dimensional Integrals by means of Finite-Dimensional approximations outside of the always used [computer oriented] Space-Time Lattice approximations (see [2], [3, chapter 9]). As a first step to tackle upon the above cited problem it will be needed to characterize mathematically the Functional Domain where these Functional Integrals are defined. 
The purpose of this paper is to present the formulation of Euclidean Quantum Field theories as Functional Fourier Transforms by means of the Bochner-Martin-Kolmogorov theorem for Topological Vector Spaces ([4], [5, Theorem 4.35]) and suitable to define and analyze rigorously Functional Integrals by means of the well-known Minlos theorem ([5, Theorem 4.312] and [6, part 2]) which is presented in full in Appendix A.

We thus present studies on the difficult problem of defining rigorously infinitedimensional quantum field path integrals in general finite volume space times $\Omega \subset R^{v}(v=$ $2,4, \ldots$ ) by means of the analytical regularization scheme [7].

\section{Some Rigorous Finite-Volume Quantum Field Path Integral in the Analytical Regularization Scheme}

Let us thus start our analysis by considering the Gaussian measure on $L^{2}\left(R^{2}\right)$ defined by the finite volume, infrared regularized, and $\alpha$-power Laplacian acting on $L^{2}\left(R^{N}\right)$ as an operatorial quadratic form $\left(j(x) \in L^{2}\left(R^{N}\right)\right.$ ) (see Appendix B)

$$
\begin{aligned}
Z_{\alpha, \varepsilon_{\mathbb{R}}}^{(0)}[j] & =\exp \left\{-\frac{1}{2}\left\langle j,\left(X_{\Omega}\left[(-\Delta)^{-\alpha}+\varepsilon_{\mathrm{IR}}^{2}\right]^{-1} X_{\Omega}\right) j\right\rangle_{L^{2}\left(R^{2}\right)}\right\} \\
& \equiv \int_{L^{2}\left(R^{2}\right)} d_{\alpha, \varepsilon_{\mathbb{R}}}^{(0)} \mu[\varphi] \exp \left(i\langle j, \varphi\rangle_{L^{2}\left(R^{2}\right)}\right) .
\end{aligned}
$$

Here $\chi_{\Omega}$ denotes the multiplication operator defined by the characteristic function $\chi_{\Omega}(x)$ of the compact region $\Omega \subset R^{2}$ and $\varepsilon_{\mathrm{IR}}>0$ the associated infrared cutoff.

It is worth calling the reader attention that due to the infrared regularization introduced on (2.1a), the domain of the Gaussian measure $[4,6]$ is given by the space of square integrable functions on $R^{2}$ by the Minlos theorem of Appendix A, since for $\alpha>1$, the operator defines a trace class operator on $L^{2}\left(R^{2}\right)$, namely,

$$
\operatorname{Tr}_{\phi_{1}\left(L^{2}\left(R^{2}\right)\right)}\left\{X_{\Omega}\left[(-\Delta)^{\alpha}+\varepsilon_{\mathrm{IR}}^{2}\right]^{-1} X_{\Omega}\right\}=\operatorname{vol}(\Omega) \times\left[\int \frac{d^{2} p}{|p|^{2 \alpha}+\varepsilon_{\mathrm{IR}}^{2}}\right]<\infty .
$$

This is the only point of our analysis where it is needed to consider the infrared cut off. As a consequence of the above remarks, one can analyze the ultraviolet renormalization program in the following interacting model proposed by us and defined by an interaction $g_{\text {bare }} V(\varphi(x))$, with $V(x)$ being the Fourier Transformed of an integrable and essentially bounded measurable real function. (It could be as well consider a polynomial interaction of the form $V_{n, p}(x)=$ minimum of $\left\{(\varphi(x))^{p}, n\right\}$ with $p$ and $n$ positive integers. Note that $\tilde{V}(k) \in L^{1}(R) \cap L^{\infty}(R)$ by hypothesis $\left|\int_{\Omega}\left[\int e^{i k \varphi(x)} \cdot \tilde{V}(k) d k\right] d^{2} x\right| \leq \operatorname{vol}(\Omega)\|\tilde{V}\|_{L^{1}}<\infty$.) Note that $V(x)$ is thus a continuous real function vanishing at the infinite point.

Let us show that by defining an ultraviolet renormalized coupling constant (with a finite volume $\Omega$ cutoff built in)

$$
g_{\text {bare }}(\alpha)=\frac{g_{\text {ren }}}{(1-\alpha)^{1 / 2}}\left(2^{-\alpha} \pi^{-1 / 4}\right)
$$


one can show that the interaction function

$$
\exp \left\{-g_{\text {bare }}(\alpha) \int_{\Omega} d^{2} x V(\varphi(x))\right\}
$$

is an integrable function on $L^{1}\left(L^{2}\left(R^{2}\right), d_{\alpha, \varepsilon_{\mathbb{R}}}^{(0)} \mu[\varphi]\right)$ and leads to a well-defined ultraviolet functional integral in the limit of $\alpha \rightarrow 1$.

The proof is based on the following estimates.

Since almost everywhere we have the pointwise limit

$$
\begin{aligned}
& \exp \left\{-g_{\text {bare }}(\alpha) \int d^{2} x V(\varphi(x))\right\} \\
& \lim _{N \rightarrow \infty}\left\{\sum_{n=0}^{N} \frac{(-1)^{n}\left(g_{\text {bare }}(\alpha)\right)^{n}}{n !} \int d k_{1} \cdots d k_{N} \tilde{V}\left(k_{1}\right) \cdots \tilde{V}\left(k_{N}\right)\right. \\
& \left.\quad \times \int_{\Omega} d x_{1} \cdots d x_{N} e^{i k_{1} \varphi\left(x_{1}\right)} \cdots e^{i k_{n} \varphi\left(x_{N}\right)}\right\}
\end{aligned}
$$

we have that the upper bound estimate below holds true

$$
\begin{aligned}
\left|Z_{\varepsilon_{\mathrm{IR}}}^{\alpha}\left[g_{\text {bare }}\right]\right| \leq & \mid \sum_{n=0}^{\infty} \frac{(-1)^{n}\left(g_{\text {bare }}(\alpha)\right)^{n}}{n !} \int_{R} d k_{1} \cdots d k_{n} \tilde{V}\left(k_{1}\right) \cdots \tilde{V}\left(k_{n}\right) \\
& \int_{\Omega} d x_{1} \cdots d x_{n} \int d_{\alpha, \varepsilon_{\mathbb{R}}}^{(0)} \mu[\varphi]\left(e^{i \sum_{\ell=1}^{N} k_{\ell} \varphi\left(x_{\ell}\right)}\right) \mid
\end{aligned}
$$

With

$$
Z_{\varepsilon_{\mathrm{R}}}^{\alpha}\left[g_{\text {bare }}\right]=\int d_{\alpha, \varepsilon_{\mathrm{R}}}^{(0)} \mu[\varphi] \exp \left\{-g_{\text {bare }}(\alpha) \int_{\Omega} d^{2} x V(\varphi(x))\right\}
$$

we have, thus, the more suitable form after realizing the $d^{2} k_{i}$ and $d_{\alpha, \varepsilon_{\mathbb{R}}}^{(0)} \mu[\varphi]$ integrals, respectively

$$
\begin{aligned}
\left|Z_{\varepsilon_{\mathbb{R}}=0}^{\alpha}\left[g_{\text {bare }}\right]\right| \leq & \sum_{n=0}^{\infty} \frac{\left(g_{\text {bare }}(\alpha)\right)^{n}}{n !}\left(\|\tilde{V}\|_{L^{\infty}(R)}\right)^{n} \\
& \times\left|\int_{\Omega} d x_{1} \cdots d x_{m} \operatorname{det}^{-1 / 2}\left[G_{\alpha}^{(m)}\left(x_{i}, x_{j}\right)\right]_{\substack{1 \leq i \leq m \\
1 \leq j \leq m}}\right| .
\end{aligned}
$$


Here $\left[G_{\alpha}^{(N)}\left(x_{i}, x_{j}\right)\right]_{1 \leq i \leq N, 1 \leq j \leq N}$ denotes the $N \times N$ symmetric matrix with the $(i, j)$ entry given by the positive Green-function of the $\alpha$-Laplacian (without the infrared cut off here!).

$$
G_{\alpha}\left(x_{i}, x_{j}\right)=\left|x_{i}-x_{j}\right|^{2(\alpha-1)} \frac{\Gamma(1-\alpha)}{\Gamma(\alpha)}\left(\frac{e^{-i \pi \alpha}}{4^{\alpha} \cdot \pi^{1 / 2}}\right) .
$$

At this point, we call the reader attention that we have the formulae on the asymptotic behavior for $\alpha \rightarrow 1$ and $\alpha<1$ (see [7, Appendix A])

$$
\begin{aligned}
\left\{\lim _{\substack{\alpha \rightarrow 1 \\
\alpha>1}} \operatorname{det}^{-1 / 2}\left[G_{\alpha}^{(N)}\left(x_{i}, x_{j}\right)\right]\right\} & \sim e^{(N / 2) \pi i \alpha} \times\left(\pi^{N / 4} \cdot 2^{N \alpha}\right) \\
& \times(1-\alpha)^{N / 2}\left(\left|\frac{(+1)}{(-1)^{N}(N-1)}\right|\right) .
\end{aligned}
$$

After substituting (2.8) into (2.6) and taking into account the hypothesis of the compact support of the nonlinearity $\tilde{V}(k)$, one obtains the finite bound for any value $g_{\text {ren }}>0$, with the finite volume cutoff and producing a proof for the convergence of the perturbative expansion in terms of the renormalized coupling constant for the model

$$
\begin{aligned}
\lim _{\alpha \rightarrow 1}\left|Z_{\varepsilon_{\mathrm{R}}=0}^{\alpha}\left[g_{\text {bare }}(\alpha)\right]\right| & \leq \sum_{n=0}^{\infty} \frac{\left(\|\tilde{V}\|_{L^{\infty}(R)}\right)^{n}}{n !}\left(\frac{g_{\text {ren }}}{(1-\alpha)^{1 / 2}}\right)^{n} \times(1-\alpha)^{n / 2} \times(\operatorname{vol}(\Omega))^{n} \\
& \leq \exp \left\{g_{\text {ren }}\|\tilde{V}\|_{L^{\infty}(R)} \operatorname{vol}(\Omega)\right\}<\infty .
\end{aligned}
$$

Another important rigorously defined functional integral is to consider the following $\alpha$-power Klein Gordon operator on Euclidean space-time (with $f \in L^{2}\left(R^{N}\right)$ )

$$
\mathcal{L}_{\Omega, f}^{-1}=\left(X_{\Omega}(2 \pi)^{v / 2}\right)\left[(-\Delta)^{\alpha}+f_{X_{\Omega}}+m^{2}\right]^{-1} \times\left(\frac{X_{\Omega}(2 \pi)^{v / 2}}{\operatorname{vol}(\Omega)}\right)
$$

with $m^{2}$ a positive "mass" parameters.

Let us note that $\mathcal{L}_{\Omega, f}^{-1}$ is an operator of trace class on $L^{2}\left(R^{v}\right)$ if and only if the result below holds true

$$
\operatorname{Tr}_{L^{2}\left(R^{v}\right)}\left(\mathcal{L}^{-1}\right)=\int d^{v} k \frac{1}{k^{2 \alpha}+m^{2}}=\bar{C}(v) m^{((v / \alpha)-2)} \times\left\{\frac{\pi}{2 \alpha} \operatorname{cosec} \frac{v \pi}{2 \alpha}\right\}<\infty,
$$

namely, if

$$
\alpha>\frac{v}{2}
$$


In this case, let us consider the double functional integral with functional domain $L^{2}\left(R^{v}\right)$

$$
\begin{aligned}
Z[j, k]= & \int d_{G}^{(0)} \beta[v(x)] \times \int d_{\mathcal{L}_{\Omega, v}}^{(0)} \mu[\varphi] \\
& \times \exp \left\{i \int d^{v} x(j(x) \varphi(x)+k(x) v(x))\right\},
\end{aligned}
$$

where the Gaussian functional integral on the fields $V(x)$ has a Gaussian generating functional defined by a $\oint_{1}$-integral operator with a positive-definite kernel $g(|x-y|)$, namely,

$$
\begin{aligned}
Z^{(0)}[k] & =\int d_{G}^{(0)} \beta[v(x)] \exp \left\{i \int d^{v} x k(x) v(x)\right\} \\
& =\exp \left\{-\frac{1}{2} \int_{\Omega} d^{v} x \int_{\Omega} d^{v} y(k(x) g(|x-y|) k(y))\right\} .
\end{aligned}
$$

A simple direct application of the Fubbini-Tonelli theorem on the exchange of the integration order on (2.13) leads us to the effective $\lambda \varphi^{4}$-(Formal) functional integral representation

$$
\begin{aligned}
Z_{\mathrm{eff}}[j]= & \int d_{\perp}^{(0)} \mu[\varphi][\varphi(x)] \\
& \times \exp \left\{-\frac{1}{2} \int_{\Omega} d^{v} x d^{v} y|\varphi(x)|^{2} g(|x-y|)|\varphi(y)|^{2}\right\} \\
& \times \exp \left\{i \int_{\Omega} d^{v} x j(x) \varphi(x)\right\} .
\end{aligned}
$$

Note that if one introduces from the beginning a bare mass parameter $m_{\text {bare }}^{2}$ depending on the parameter $\alpha$, but such that it always satisfies (2.11) one should obtain again (2.15) as a well-defined measure on $L^{2}\left(R^{v}\right)$. Of course that the usual pure Laplacian limit of $\alpha \rightarrow 1$ on (2.10), there will be needed a renormalization of this mass parameters $\left(\lim _{\alpha \rightarrow 1} m_{\text {bare }}^{2}(\alpha)=\right.$ $+\infty$ !) as much as it has been done in previous studies.

Let us continue our examples by showing again the usefulness of the precise determination of the functional-distributional structure of the domain of the functional integrals in order to construct rigorously these path integrals without complicated lattice limit procedures [2].

Let us consider a general $R^{v}$ Gaussian measure defined by the Generating functional on $S\left(R^{v}\right)$ defined by the $\alpha$-power of the Laplacian operator $-\Delta$ acting on $S\left(R^{v}\right)$ with a small infrared regularization mass parameter $\mu^{2}$ as considered in (2.1a)

$$
\begin{aligned}
Z_{(0)}[j] & =\exp \left\{-\frac{1}{2}\left\langle j,\left((-\Delta)^{\alpha}+\mu_{0}^{2}\right)^{-1} j\right\rangle_{L^{2}\left(R^{v}\right)}\right\} \\
& =\int_{E^{\mathrm{alg}}\left(S\left(R^{v}\right)\right)} d_{\alpha}^{(0)} \mu[\varphi] \exp (i \varphi(j)) .
\end{aligned}
$$


An explicit expression in momentum space for the Green function of the $\alpha$-power of $(-\Delta)^{\alpha}+\mu_{0}^{2}$ given by

$$
\left((-\Delta)^{+\alpha}+\mu_{0}^{2}\right)^{-1}(x-y)=\int \frac{d^{v} k}{(2 \pi)^{v}} e^{i k(x-y)}\left(\frac{1}{k^{2 \alpha}+\mu_{0}^{2}}\right) .
$$

Here $\bar{C}(v)$ is a $v$-dependent (finite for $v$-values !) normalization factor.

Let us suppose that there is a range of $\alpha$-power values that can be choosen in such way that one satisfies the constraint below

$$
\int_{E^{\mathrm{alg}\left(S\left(R^{v}\right)\right)}} d_{\alpha}^{(0)} \mu[\varphi]\left(\|\varphi\|_{L^{2 j}\left(R^{v}\right)}\right)^{2 j}<\infty
$$

with $j=1,2, \ldots, N$ and for a given fixed integer $N$, the highest power of our polynomial field interaction. Or equivalently, after realizing the $\varphi$-Gaussian functional integration, with a space-time cuttoff volume $\Omega$ on the interaction to be analyzed on (2.16)

$$
\begin{aligned}
\left|\int_{\Omega} d^{v} x\left[(-\Delta)^{\alpha}+\mu_{0}^{2}\right]^{-j}(x, x)\right| & \leq\left|\operatorname{vol}(\Omega) \times\left(\int \frac{d^{v} k}{k^{2 \alpha}+\mu_{0}^{2}}\right)^{j}\right| \\
& =\left|\left[C_{v}\left(\mu_{0}\right)^{((v / \alpha)-2)} \times\left(\frac{\pi}{2 \alpha} \operatorname{cosec} \frac{v \pi}{2 \alpha}\right)\right]^{j} \times \operatorname{vol}(\Omega)\right|<\infty .
\end{aligned}
$$

For $\alpha>v / 2$, one can see by the Minlos theorem that the measure support of the Gaussian measure (2.16) will be given by the intersection Banach space of measurable Lebesgue functions on $R^{v}$ instead of the previous one $E^{\mathrm{alg}}\left(S\left(R^{v}\right)\right)([4-6])$

$$
\mathcal{L}_{2 N}\left(R^{v}\right)=\bigcap_{j=1}^{N}\left(L^{2 j}\left(R^{v}\right)\right)
$$

In this case, one obtains that the finite-volume $p(\varphi)_{2}$ interactions

$$
\exp \left\{-\sum_{j=1}^{N} \lambda_{2 j} \int_{\Omega}\left(\varphi^{2}(x)\right)^{j} d x\right\} \leq 1
$$

are mathematically well defined as the usual pointwise product of measurable functions and for positive coupling constant values $\lambda_{2 j} \geq 0$. As a consequence, we have a measurable functional on $L^{1}\left(\mathcal{L}_{2 N}\left(R^{v}\right) ; d_{\alpha}^{(0)} \mu[\varphi]\right)$ (since it is bounded by the function 1 ). Thus, it makes sense to consider mathematically the well-defined path integral on the full space $R^{v}$ with those values of the power $\alpha$ satisfying the constraint (2.17).

$$
Z[j]=\int_{\mathcal{L}_{2 N}\left(R^{v}\right)} d_{\alpha}^{(0)} \mu[\varphi] \exp \left\{-\sum_{j=1}^{N} \lambda_{2 j} \int_{\Omega} \varphi^{2 j}(x) d x\right\} \times \exp \left(i \int_{R^{v}} j(x) \varphi(x)\right) .
$$


Finally, let us consider an interacting field theory in a compact space-time $\Omega \subset R^{v}$ defined by an iteger even power $2 n$ of the Laplacian operator with Dirichlet Boundary conditions as the free Gaussian kinetic action, namely,

$$
\begin{aligned}
Z^{(0)}[j] & =\exp \left\{-\frac{1}{2}\left\langle j,(-\Delta)^{-2 n} j\right\rangle_{L^{2}(\Omega)}\right\} \\
& =\int_{W_{2}^{n}(\Omega)} d_{(2 n)}^{(0)} \mu[\varphi] \exp \left(i\langle j, \varphi\rangle_{L^{2}(\Omega)}\right)
\end{aligned}
$$

here $\varphi \in W_{2}^{n}(\Omega)$-the Sobolev space of order $n$ which is the functional domain of the cylindrical Fourier Transform measure of the Generating functional $Z^{(0)}[j]$, a continuous bilinear positive form on $W_{2}^{-n}(\Omega)$ (the topological dual of $W_{2}^{n}(\Omega)$ ) [4-6].

By a straightforward application of the well-known Sobolev immersion theorem, we have that for the case of

$$
n-k>\frac{v}{2}
$$

including $k$ as a real number the functional Sobolev space $W_{2}^{n}(\Omega)$ is contained in the continuously fractional differentiable space of functions $C^{k}(\Omega)$. As a consequence, the domain of the Bosonic functional integral can be further reduced to $C^{k}(\Omega)$ in the situation of (2.24)

$$
Z^{(0)}[j]=\int_{C^{k}(\Omega)} d_{(2 n)}^{(0)} \mu[\varphi] \exp \left(i\langle j, \varphi\rangle_{L^{2}(\Omega)}\right)
$$

That is our new result generalizing the Wiener theorem on Brownian paths in the case of $n=1, k=1 / 2$, and $v=1$.

Since the bosonic functional domain on (2.25) is formed by real functions and not distributions, we can see straightforwardly that any interaction of the form

$$
\exp \left\{-g \int_{\Omega} F(\varphi(x)) d^{v} x\right\}
$$

with the nonlinearity $F(x)$ denoting a lower bounded real function $(\gamma>0)$

$$
F(x) \geq-\gamma
$$

is well-defined and is integrable function on the functional space $\left(C^{k}(\Omega), d_{(2 n)}^{(0)} \mu[\varphi]\right)$ by a direct application of the Lebesgue theorem

$$
\left|\exp \left\{-g \int_{\Omega} F(\varphi(x)) d^{v} x\right\}\right| \leq \exp \{+g \gamma\}
$$

At this point we make a subtle mathematical remark that the infinite volume limit of (2.25)-(2.26) is very difficult, since one looses the Garding-Poincaré inequality at this limit for 
those elliptic operators and, thus, the very important Sobolev theorem. The probable correct procedure to consider the thermodynamic limit in our Bosonic path integrals is to consider solely a volume cutoff on the interaction term Gaussian action as in (2.22) and there search for $\operatorname{vol}(\Omega) \rightarrow \infty[8-11]$.

As a last remark related to (2.23) one can see that a kind of "fishnet" exponential generating functional

$$
Z^{(0)}[j]=\exp \left\{-\frac{1}{2}\langle j, \exp \{-\alpha \Delta\} j\rangle_{L^{2}(\Omega)}\right\}
$$

has a Fourier transformed functional integral representation defined on the space of the infinitely differentiable functions $C^{\infty}(\Omega)$, which physically means that all field configurations making the domain of such path integral has a strong behavior like purely nice smooth classical field configurations.

As a last important point of this paper, we present an important result on the geometrical characterization of massive free field on an Euclidean Space-Time [11].

Firstly we announce a slightly improved version of the usual Minlos Theorem [4].

Theorem 2.1. Let E be a nuclear space of tests functions and $d \mu$ a given $\sigma$-measure on its topological dual with the strong topology. Let $\langle,\rangle_{0}$ be an inner product in $E$, inducing a Hilbertian structure on $\mathscr{H}_{0}=\overline{\left.(E, \backslash,\rangle_{0}\right)}$, after its topological completion.

We suppose the following.

(a) There is a continuous positive definite functional in $\mathscr{\ell}_{0}, Z(j)$, with an associated cylindrical measure $d \mu$.

(b) There is a Hilbert-Schmidt operator $T: \mathscr{l}_{0} \rightarrow \mathscr{L}_{0}$; invertible, such that $E \subset$ Range $(T), T^{-1}(E)$ is dense in $\mathscr{\ell}_{0}$ and $T^{-1}: \mathscr{\ell}_{0} \rightarrow \mathscr{\ell}_{0}$ is continuous.

We have thus, that the support of the measure satisfies the relationship

$$
\text { support } d \mu \subseteq\left(T^{-1}\right)^{*}\left(\mathscr{\ell}_{0}\right) \subset E^{*} \text {. }
$$

At this point we give a nontrivial application of ours of the above cited Theorem 2.1.

Let us consider a differential inversible operator $\mathcal{L}: S^{\prime}\left(R^{N}\right) \rightarrow S(R)$, together with a positive inversible self-adjoint elliptic operator $P: D(P) \subset L^{2}\left(R^{N}\right) \rightarrow L^{2}\left(R^{N}\right)$. Let $H_{\alpha}$ be the following Hilbert space:

$$
H_{\alpha}=\left\{\overline{S\left(R^{N}\right),\left\langle P^{\alpha} \varphi, P^{\alpha} \varphi\right\rangle_{L^{2}\left(R^{N}\right)}=\langle,\rangle_{\alpha}}, \text { for } \alpha \text { a real number }\right\} .
$$


We can see that for $\alpha>0$, the operators below

$$
\begin{gathered}
P^{-\alpha}: L^{2}\left(R^{N}\right) \longrightarrow \mathscr{L}_{+\alpha} \\
\varphi \longrightarrow\left(P^{-\alpha} \varphi\right), \\
P^{\alpha}: \mathscr{L}_{+\alpha} \longrightarrow L^{2}\left(R^{N}\right) \\
\varphi \longrightarrow\left(P^{\alpha} \varphi\right)
\end{gathered}
$$

are isometries among the following subspaces:

$$
\overline{\left.D\left(P^{-\alpha}\right),\langle,\rangle_{L^{2}}\right)}, H_{+\alpha}
$$

since

$$
\begin{gathered}
\left\langle P^{-\alpha} \varphi, P^{-\alpha} \varphi\right\rangle_{\mathscr{d}_{+\alpha}}=\left\langle P^{\alpha} P^{-\alpha} \varphi, P^{\alpha} P^{-\alpha} \varphi\right\rangle_{L^{2}\left(R^{N}\right)}=\langle\varphi, \varphi\rangle_{L^{2}\left(R^{N}\right)}, \\
\left\langle P^{\alpha} f, P^{\alpha} f\right\rangle_{L^{2}\left(R^{N}\right)}=\langle f, f\rangle_{H_{+\alpha}} .
\end{gathered}
$$

If one considers $T$ a given Hilbert-Schmidt operator on $H_{\alpha}$, the composite operator $T_{0}=P^{\alpha} T P^{-\alpha}$ is an operator with domain being $D\left(P^{-\alpha}\right)$ and its image being the Range $\left(P^{\alpha}\right) . T_{0}$ is clearly an invertible operator and $S\left(R^{N}\right) \subset$ Range $(T)$ means that the equation $\left(T P^{-\alpha}\right)(\varphi)=$ $f$ has always a nonzero solution in $D\left(P^{-\alpha}\right)$ for any given $f \in S\left(R^{N}\right)$. Note that the condition that $T^{-1}(f)$ be a dense subset on Range $\left(P^{-\alpha}\right)$ means that

$$
\left\langle T^{-1} f, P^{-\alpha} \varphi\right\rangle_{L^{2}\left(R^{N}\right)}=0
$$

has as unique solution the trivial solution $f \equiv 0$.

Let us suppose too that $T^{-1}: S\left(R^{N}\right) \rightarrow H_{\alpha}$ be a continuous application and the bilinear term $\left(\mathcal{L}^{-1}(j)\right)(j)$ be a continuous application in the Hilbert spaces $H_{+\alpha} \supset S\left(R^{N}\right)$, namely, if $j_{n} \stackrel{L^{2}}{\rightarrow} j$, then $\mathcal{L}^{-1}: P^{-\alpha} j_{n} \stackrel{L^{2}}{\rightarrow} \mathcal{L}^{-1} P^{-\alpha} j$, for $\left\{j_{n}\right\}_{n \in \mathbb{Z}}$ and $\left(f_{n}, j\right) \in S\left(R^{N}\right)$.

By a direct application of the Minlos Theorem, we have the result

$$
Z(j)=\exp \left\{-\frac{1}{2}\left[\mathcal{L}^{-1}(j)(j)\right]\right\}=\int_{\left(T^{-1}\right)^{*} H_{\alpha}} d \mu(T) \exp (i T(j))
$$

Here the topological space support is given by

$$
\begin{aligned}
\left(T^{-1}\right)^{*} \mathscr{H}_{\alpha} & =\left[\left(P^{-\alpha} T_{0} P^{\alpha}\right)^{-1}\right]^{*}\left(\overline{\left(P^{\alpha}\left(S\left(R^{N}\right)\right)\right)}\right) \\
& =\left[\left(P^{\alpha}\right)^{*}\left(T_{0}^{-1}\right)^{*}\left(P^{-\alpha}\right)^{*}\right] P^{\alpha}\left(S\left(R^{N}\right)\right) \\
& =P^{\alpha} T_{0}^{-1}\left(L^{2}\left(R^{N}\right)\right) .
\end{aligned}
$$


In the important case of $\mathcal{L}=\left(-\Delta+m^{2}\right): S^{\prime}\left(R^{N}\right) \rightarrow S\left(R^{N}\right)$ and $T_{0} T_{0}^{*}=\left(\chi_{\Omega}(2 \pi)^{N / 2} /\right.$ $\operatorname{vol}(\Omega))\left(-\Delta+m^{2}\right)^{-2 \beta} \times \chi_{\Omega} \in \oint_{1}\left(L^{2}\left(R^{N}\right)\right)$ since $\operatorname{Tr}\left(T_{0} T_{0}^{*}\right)=\left(1 / 2\left(m^{2}\right)^{\beta}\right)\left(m^{2} / 1\right)^{N / 2} \Gamma(N / 2) \Gamma(2 \beta-$ $(N / 2)) / \Gamma(\beta)<\infty$ for $\beta>N / 4$ with the choice $P=\left(-\Delta+m^{2}\right)$, we can see that the support of the measure in the path-integral representation of the Euclidean measure field in $R^{N}$ may be taken as the measurable subset below

$$
\operatorname{supp}\left\{d_{\left(-\Delta+m^{2}\right)} \mu(\varphi)\right\}=\left(-\Delta+m^{2}\right)^{+\alpha} I_{\Omega}(x)\left(-\Delta+m^{2}\right)^{+\beta}\left(L^{2}\left(R^{N}\right)\right)
$$

since $\mathcal{L}^{-1} P^{-\alpha}=\left(-\Delta+m^{2}\right)^{-1-\alpha}$ is always a bounded operator in $L^{2}\left(R^{N}\right)$ for $\alpha>-1$.

As a consequence each field configuration can be considered as a kind of "fractional distributional" derivative of a square integrable function as written below of the formal infinite volume $\Omega \rightarrow R^{N}$.

$$
\varphi(x)=\left[\left(-\Delta+m^{2}\right)^{(N / 4)+\varepsilon-1} f\right](x)
$$

with a function $f(x) \in L^{2}\left(R^{N}\right)$ and any given $\varepsilon>0$, even if originally all fields configurations entering into the path integral were elements of the Schwartz Tempered Distribution Spaces $S^{\prime}\left(R^{N}\right)$ certainly very "rough" mathematical objects to characterize from a rigorous geometrical point of view.

We have, thus, made a further reduction of the functional domain of the free massive Euclidean scalar field of $S^{\prime}\left(R^{N}\right)$ to the measurable subset as given by (2.38) denoted by $W\left(R^{N}\right)$

$$
\begin{aligned}
\exp \left\{-\frac{1}{2}\left[\left(-\Delta+m^{2}\right)^{-1} j\right](j)\right\} & =\int_{S^{\prime}\left(R^{N}\right)} d_{\left(-\Delta+m^{2}\right)} \mu(\varphi) e^{i \varphi(j)} \\
& =\int_{W\left(R^{N}\right) \subset S^{\prime}\left(R^{N}\right)} d_{\left(-\Delta+m^{2}\right)} \tilde{\mu}(f) e^{i\left\langle f,\left(-\Delta+m^{2}\right)^{(N / 4)+\varepsilon-1} f\right\rangle_{L^{2}\left(R^{N}\right)}}
\end{aligned}
$$

\section{Appendices}

\section{A. Some Comments on the Support of Functional Measures in Hilbert Space}

Let us comment further on the application of the Minlos Theorem in Hilbert Spaces. In this case one has a very simple proof which holds true in general Banach Spaces $(E,\|\|)$.

Let us thus, give a cylindrical measures $d^{\infty} \mu(x)$ in the algebraic dual $E^{\text {alg }}$ of a given Banach Space E [4-6].

Let us suppose either that the function $\|x\|$ belongs to $L^{1}\left(E^{\mathrm{alg}}, d^{\infty} \mu(x)\right)$. Then the support of this cylindrical measures will be the Banach Space $E$.

The proof is the following.

Let $A$ be a subset of the vectorial space $E^{\text {alg }}$ (with the topology of pontual convergence), such that $A \subset E^{c}$ (so $\left.\|x\|=+\infty\right)$ ( $E$ can always be imbeded as a cylindrical measurable subset of $E^{\mathrm{alg}}$-just use a Hammel vectorial basis to see that). Let be the sets 
$A_{n}=\left\{x \in E^{\text {alg }} \mid\|x\| \geq n\right\}$. Then we have the set inclusion $A \subset \bigcap_{n=0}^{\infty} A_{n}$, so its measure satisfies the estimates below:

$$
\begin{aligned}
\mu(A) & \leq \liminf _{n} \mu\left(A_{n}\right) \\
& =\liminf _{n} \mu\left\{x \in E^{\mathrm{alg}} \mid\|x\| \geq n\right\} \\
& \leq \liminf _{n}\left\{\frac{1}{n} \int_{E^{\mathrm{alg}}}\|x\| d^{\infty} \mu(x)\right\} \\
& =\liminf _{n} \frac{\|x\|_{L^{1}\left(E^{\mathrm{ag},}, d_{\mu}^{\infty}\right)}}{n}=0 .
\end{aligned}
$$

Leading us to the Minlos theorem that the support of the cylindrical measure in $E^{\mathrm{alg}}$ is reduced to the own Banach Space $E$.

Note that by the Minkowisky inequality for general integrals, we have that $\|x\|^{2} \in$ $L^{1}\left(E^{\mathrm{alg}}, d^{\infty} \mu(x)\right)$. Now it is elementary evaluation to see that if $A^{-1} \in \oint_{1}(\mathcal{M})$, when $E=\mathcal{M}$, a given Hilbert Space, we have that

$$
\int_{\mathcal{M}^{\mathrm{alg}}} d_{A}^{\infty} \mu(x) \cdot\|x\|^{2}=\operatorname{Tr}_{\mathcal{M}}\left(A^{-1}\right)<\infty
$$

This result produces another criterium for supp $d_{A}^{\infty} \mu=\mathcal{M}$ (the Minlos Theorem), when $E=\mathcal{M}$ is a Hilbert Space.

It is easy too to see that if

$$
\int_{\mathcal{M}}\|x\| d^{\infty} \mu(x)<\infty
$$

then the Fourier-Transformed functional

$$
Z(j)=\int_{\mathcal{M}} e^{i(j, x)_{\mu}} d^{\infty} \mu(x)
$$

is continuous in the norm topology of $\mathcal{M}$.

Otherwise, if $Z(j)$ is not continuous in the origin $0 \in \mathcal{M}$ (without loss of generality), then there is a sequence $\left\{j_{n}\right\} \in \mathcal{M}$ and $\delta>0$, such that $\left\|j_{n}\right\| \rightarrow 0$ with

$$
\begin{aligned}
\delta \leq\left|Z\left(j_{n}\right)-1\right| & \leq \int_{\mathcal{M}}\left|e^{i\left(j_{n}, x\right)_{\mathcal{M}}}-1\right| d^{\infty} \mu(x) \\
& \leq \int_{\mathcal{M}}\left|j_{n}, x\right| d^{\infty} \mu(x) \\
& \leq\left\|j_{n}\right\|\left(\int_{\mathcal{M}}\|x\| d^{\infty} \mu(x)\right) \longrightarrow 0,
\end{aligned}
$$

a contradiction with $\delta>0$. 
Finally, let us consider an elliptic operator $B$ (with inverse) from the Sobelev space $W^{-2 m}(\Omega)$ to $W^{2 m}(\Omega)$. Then by the criterium given by (A.2) if

$$
\operatorname{Tr}_{L^{2}(\Omega)}\left[(I+\Delta)^{+m / 2} B^{-1}(I+\Delta)^{+m / 2}\right]<\infty,
$$

we will have that the path integral written below is well defined for $x \in W^{+2 m}(\Omega)$ and $j \in$ $W^{-2 m}(\Omega)$. Namely,

$$
\exp \left(-\frac{1}{2}\left(j, B^{-1} j\right)_{L^{2}(\Omega)}\right)=\int_{W^{2 m}(\Omega)} d_{B} \mu(x) \exp \left(i(j, x)_{L^{2}(\Omega)}\right) .
$$

By the Sobolev theorem which means that the embeded below is continuous (with $\Omega \subseteq R^{v}$ denoting a smooth domain), one can further reduce the measure support to the Hölder $\alpha$ continuous function in $\Omega$ if $2 m-(v / 2)>\alpha$. Namely, we have an easy proof of the famous Wiener Theorem on sample continuity of certain path integrals in Sobolev Spaces

$$
W^{2 m}(\Omega) \subset C^{\alpha}(\Omega) .
$$

The above Wiener Theorem is fundamental in order to construct nontrivial examples of mathematically rigorous euclidean's path integrals in spaces $R^{v}$ of higher dimensionality, since it is a trivial consequence of the Lebesgue theorem that positive continuous functions $V(x)$ generate functionals integrable in $\left\{W^{2 m}(\Omega), d_{B} \mu(\varphi)\right\}$ of the form below

$$
\exp \left\{-\int_{\Omega} V(\varphi(x)) d x\right\} \in L^{1}\left(W^{2 m}(\Omega), d_{B} \mu(\varphi)\right)
$$

As a last important remark on Cylindrical Measures in Separable Hilbert Spaces, let us point to our reader that the support of such above measures is always a $\sigma$-compact set in the norm topology of $\mathcal{M}$. In order to see such result let us consider a given dense set of $\mathcal{M}$, namely, $\left\{x_{k}\right\}_{k \in I^{+}}$. Let $\left\{\delta_{k}\right\}_{k \in I^{+}}$be a given sequence of positive real numbers with $\delta_{k} \rightarrow 0$. Let $\left\{\varepsilon_{n}\right\}$ be another sequence of positive real numbers such that $\sum_{n=1}^{\infty} \varepsilon_{n}<\varepsilon$. Now it is straightforward to see that $\mathcal{M} \subset \bigcup_{h=1}^{\infty} \overline{B\left(x_{k}, \delta_{k}\right)} \subset \mathcal{M}$ and thus $\lim \sup \mu\left\{\bigcup_{k=1}^{n} \overline{B\left(x_{k}, \delta_{k}\right)}\right\}=\mu(\mathcal{M})=1$. As a consequence, for each $n$, there is a $k_{n}$, such that $\mu\left(\bigcup_{k=1}^{k_{n}} \overline{B\left(x_{k}, \delta_{k}\right)}\right) \geq 1-\varepsilon$.

Now the sets $K_{\mu}=\bigcap_{n=1}^{\infty}\left[\bigcup_{k=1}^{k_{n}} \overline{B\left(x_{k}, \delta_{k}\right)}\right]$ are closed and totally bounded, so they are compact sets in $\mathcal{M}$ with $\mu(\mathcal{M}) \geq 1-\varepsilon$. Let us now choose $\varepsilon=1 / n$ and the associated compact sets $\left\{K_{n, \mu}\right\}$. Let us further consider the compact sets $\widehat{K}_{n, \mu}=\bigcup_{\ell=1}^{n} K_{\ell, \mu}$. We have that $\widehat{K}_{n, \mu} \subseteq$ $\widehat{K}_{n+1, \mu}$, for any $n$ and $\lim \sup \mu\left(\widehat{K}_{n, \mu}\right)=1$. So, Supp $d \mu=\bigcup_{n=1}^{\infty} \widehat{K}_{n, \mu}$, a $\sigma$-compact set of $\mathcal{M}$.

We consider now a enumerable family of cylindrical measures $\left\{d \mu_{n}\right\}$ in $\mathcal{M}$ satisfying the chain inclusion relationship for any $n \in I^{+}$

$$
\operatorname{Supp} d \mu_{n} \subseteq \operatorname{Supp} d \mu_{n+1} \text {. }
$$

Now it is straightforward to see that the compact sets $\left\{\widehat{K}_{n}^{(n)}\right\}$, where Supp $d \mu_{m}=\bigcup_{n=1}^{\infty} \widehat{K}_{n}^{(m)}$, is such that Supp $\left\{d \mu_{m}\right\} \subseteq \bigcup_{n=1}^{\infty} \widehat{K}_{n}^{(n)}$, for any $m \in I^{+}$. 
Let us consider the family of functionals induced by the restriction of this sequence of measures in any compact $\widehat{K}_{n}^{(n)}$. Namely,

$$
\mu_{n} \longrightarrow L_{n}^{(n)}(f)=\int_{\widehat{K}_{n}^{(n)}} f(x) \cdot d \mu_{p}(x)
$$

Here $f \in C_{b}\left(\widehat{K}_{n}^{(n)}\right)$. Note that all the above functionals in $\bigcup_{n=1}^{\infty} C_{b}\left(\widehat{K}_{n}^{(n)}\right)$ are bounded by 1 . By the Alaoglu-Bourbaki theorem they form a compact set in the weak star topology of $\left(\bigcup_{n=1}^{\infty} C_{b}\left(\widehat{K}_{n}^{(n)}\right)\right)^{*}$, so there is a subsequence (or better the whole sequence) converging to a unique cylindrical measure $\bar{\mu}(x)$. Namely,

$$
\lim _{n \rightarrow \infty} \int_{\mathcal{M}} f(x) d \mu_{n}(x)=\int_{\mathcal{M}} f(x) d \bar{\mu}(x)
$$

for any $f \in \bigcup_{n=1}^{\infty} C_{b}\left(\widehat{K}_{n}^{(n)}\right)$.

\section{B. On the Trace Class Property}

A straightforward calculation gives the following expression for the trace of the integral operator on (2.1a)

$$
\operatorname{Tr}_{\phi_{1}}\left\{X_{\Omega}\left[(-\Delta)^{\alpha}+\varepsilon_{\mathrm{IR}}^{2}\right]^{-1} X_{\Omega}\right\}=\int_{-\infty}^{+\infty}\left\{\int_{-\infty}^{+\infty}\left[\frac{\tilde{X}_{\Omega}(k-p) \tilde{X}_{\Omega}(p-k)}{\left(p^{2 \alpha}+\varepsilon_{\mathrm{IR}}^{2}\right)}\right] d p\right\} d k,
$$

where

$$
\tilde{X}_{\Omega}(p)=\frac{1}{2 \pi}\left\{\int_{-\infty}^{+\infty} X_{\Omega}(x) e^{i p x} d^{2} x\right\}
$$

Since by Parserval's theorem

$$
\int_{-\infty}^{+\infty} d k\left|\tilde{X}_{\Omega}(k-p)\right|^{2} d^{2} k=\int_{-\infty}^{+\infty}\left|\chi_{\Omega}(x)\right|^{2} d^{2} x=\operatorname{vol}(\Omega)
$$

one has the validity of the result written in $(2.1 \mathrm{~b})$.

\section{References}

[1] B. Simon, Functional Integration and Quantum Physics, vol. 86 of Pure and Applied Mathematics, Academic Press, New York, NY, USA, 1979.

[2] B. Simon, The $P(\phi)_{2}$ Euclidean (Quantum) Field Theory, Princeton University Press, Princeton, NJ, USA, 1974.

[3] J. Glimm and A. Jaffe, Quantum Physics, Springer, New York, NY, USA, 2nd edition, 1987.

[4] Y. Yamasaki, Measures on Infinite-Dimensional Spaces, vol. 5 of Series in Pure Mathematics, World Scientific Publishing, Singapore, 1985. 
[5] D. X. Xia, Measure and Integration Theory on Infinite-Dimensional Spaces. Abstract Harmonic Analysis, Academic Press, New York, NY, USA, 1972.

[6] L. Schwartz, Radon Measures on Arbitrary Topological Spaces and Cylindrical Measures, Tata InstituteOxford University Press, London, UK, 1973.

[7] L. L. C. Botelho, "A simple renormalization scheme in random surface theory," Modern Physics Letters B, vol. 13, no. 6-7, pp. 203-207, 1999.

[8] K. Symanzik, "Euclidean quantum field theory. I. Equations for a scalar model," Journal of Mathematical Physics, vol. 7, pp. 510-525, 1966.

[9] B. Simon, "Distributions and their hermite expansions," Journal of Mathematical Physics, vol. 12, no. 1, pp. 140-148, 1971.

[10] L. C.L. Botelho, "Path-integral bosonization for massive fermion models in two dimensions," Physical Review D, vol. 33, no. 4, pp. 1195-1198, 1986.

[11] E. Nelson, "Regular probability measures on function space," Annals of Mathematics. Second Series, vol. 69, pp. 630-643, 1959. 


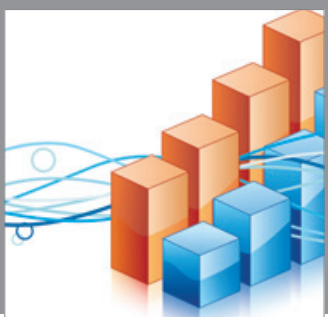

Advances in

Operations Research

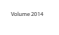

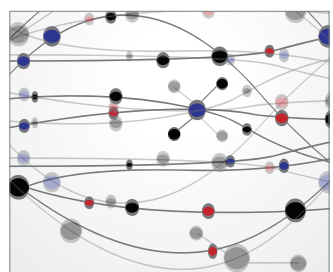

\section{The Scientific} World Journal
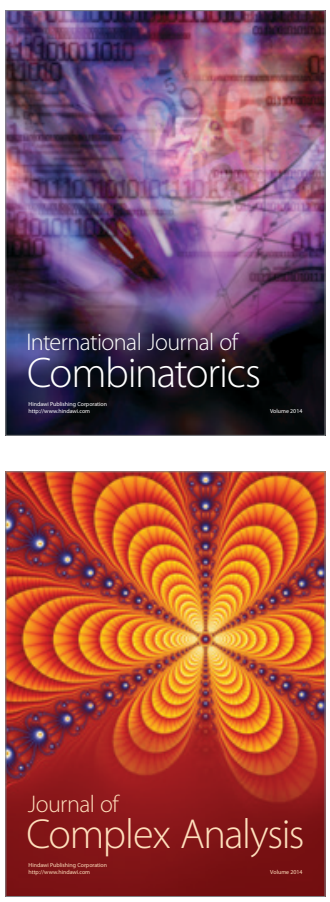

International Journal of

Mathematics and

Mathematical

Sciences
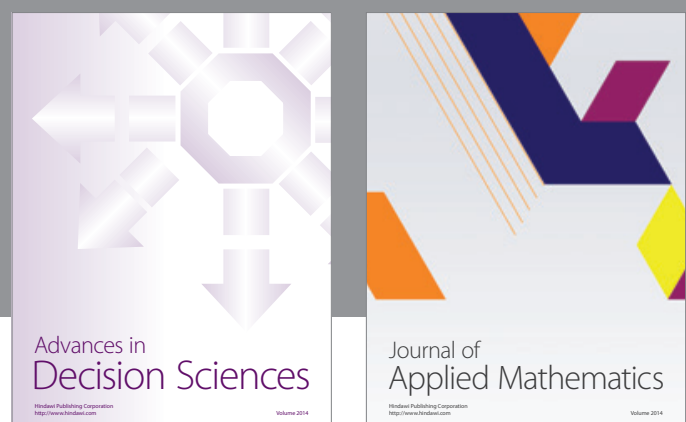

Journal of

Applied Mathematics
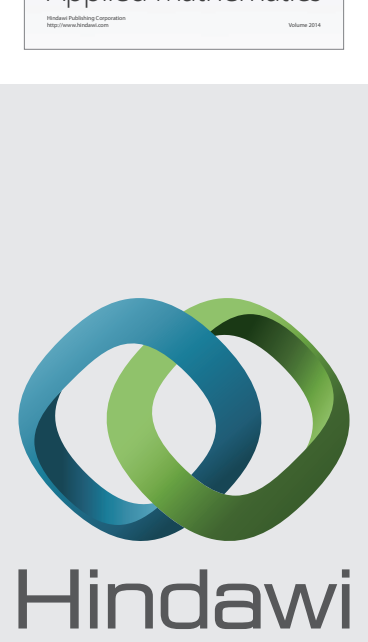

Submit your manuscripts at http://www.hindawi.com
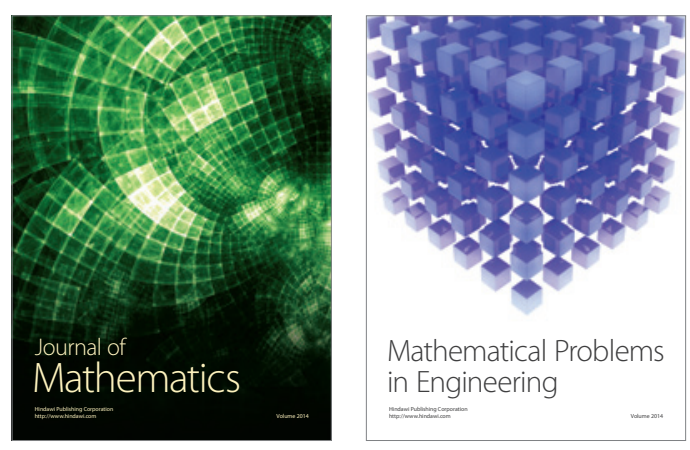

Mathematical Problems in Engineering
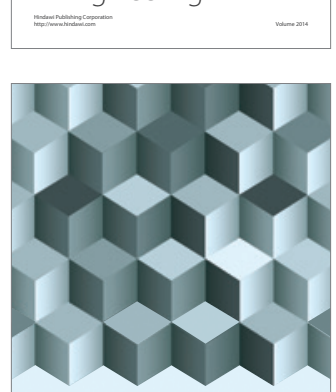

Journal of

Function Spaces
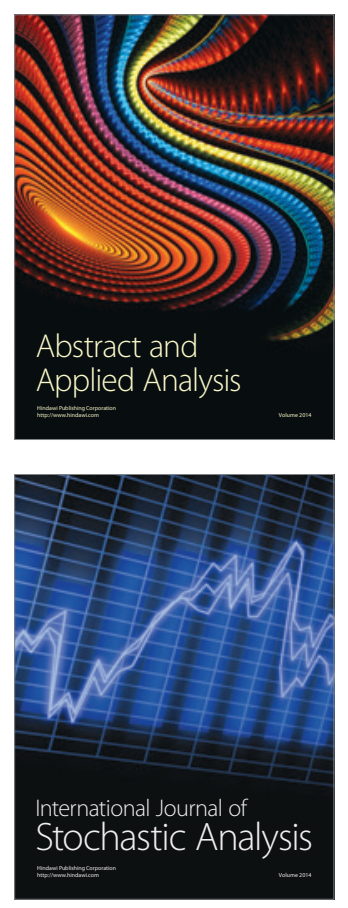

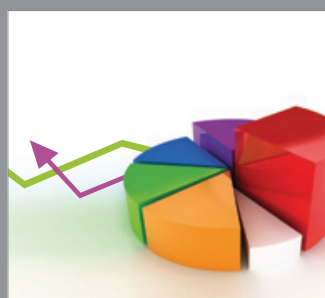

ournal of

Probability and Statistics

Promensencen
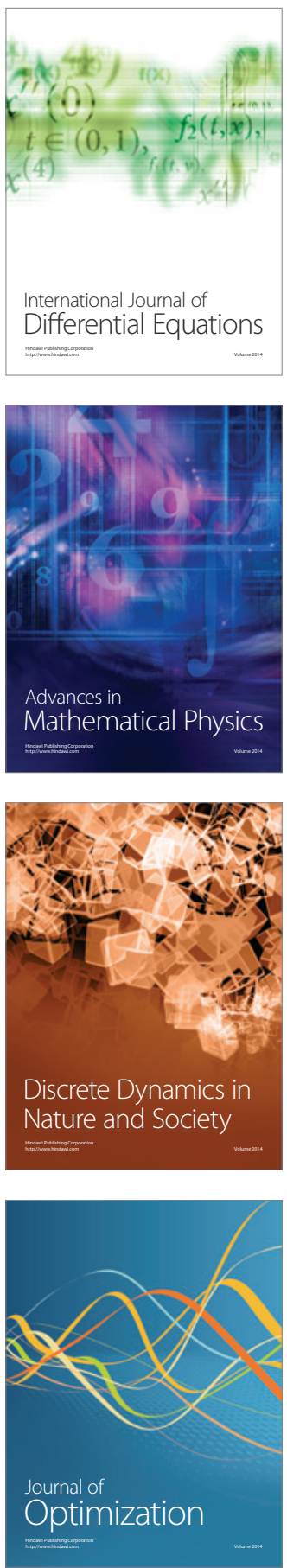